\title{
Induction of Haemeoxygenase-1 Directly Improves Endothelial Function in Isolated Aortas from Obese Rats through the Ampk-Pi3k/Akt-Enos Pathway
}

\author{
Fang Han ${ }^{a}$ Ying Guo ${ }^{b}$ Lili Xuc Ningning Hou ${ }^{\mathrm{b}}$ Fei Han ${ }^{\mathrm{b}}$ Xiaodong Sun \\ a Department of Pathology, ${ }^{b}$ Department of Endocrinology, Affiliated Hospital of Weifang Medical \\ University, Weifang, 'Department of Endocrinology, Affiliated Hospital of Qingdao University, Qingdao, \\ China
}

\section{Key Words}

Haemeoxygenase-1 1 Free fatty acids • Endothelial dysfunction

\begin{abstract}
Background: Induction of haemeoxygenase-1 (HO-1) increases adiponectin secretion by remodeling adipose tissue in obesity. The objective of our study is to explore whether HO-1 induction directly improves endothelial function independent of adiponectin changes in obese rats. Methods: Rats were divided into control and obesity groups. Aortic endothelial function was determined by measuring endothelium-dependent vasodilatation (EDV). Vascular segments of the obese rats were incubated in an organ bath in the presence or absence of cobalt protoporphyrin (CoPP) or CoPP plus stannous protoporphyrin. Nitric oxide (NO) production, superoxide anion production and NF-KB p65 expression in the aorta were determined. The expression of AMP-activated kinase (AMPK), Akt and endothelial nitric oxide synthase (eNOS) in endothelial cells was determined by western blotting. The aortic rings from the obese rats were then incubated with CoPP in the presence of specific inhibitors of AMPK, phosphatidylinositol 3-kinase (PI3K) or eNOS. Results: Acetylcholine-induced EDV was significantly attenuated in the obese rats, compared with the NC group $(p<0.05)$. Preincubation of vessels from obese rats with CoPP significantly increased EDV $(p<0.05)$. However, this beneficial effect of CoPP was partly attenuated in vitro in the presence of inhibitors of AMPK, PI3K or eNOS. HO-1 induction with CoPP significantly increased the activation of the AMPK-PI3K/Akt-eNOS pathway and NO production in parallel with reduced superoxide anion production and NF-KB p65 expression in obese rats. Conclusions: $\mathrm{HO}-1$ induction with CoPP directly improved endothelial function in obese rats independent of adiponectin changes. The mechanism of this protective effect is related to increasing NO production by activation of the AMPK-PI3K/Akt-eNOS signaling pathway.
\end{abstract}

Xiaodong Sun

KARGER 125
Department of Endocrinology, Affiliated Hospital of Weifang Medical University, No.2428, Yuhe Road, Weifang, Shandong, 261031 (China)

Tel. +86-18653625906, E-Mail sxdfriend@sina.com 


\begin{tabular}{|c|c|c|}
\hline Cellular Physiology & Cell Physiol Biochem 2015;36:1480-1490 & \\
\hline and Biochemistry & $\begin{array}{l}\text { DOI: 10.1159/000430312 } \\
\text { Published online: July 06, } 2015\end{array}$ & $\begin{array}{l}\text { O } 2015 \text { S. Karger AG, Basel } \\
\text { www.karger.com/cpb }\end{array}$ \\
\hline
\end{tabular}

\section{Introduction}

The haemeoxygenase-1 (HO) system is the primary pathway present in many tissues to catalyze the oxidation of heme, a potentially harmful pro-oxidant, to the biologically active molecules biliverdin, iron and carbon monoxide (CO) [1, 2]. To date, two main distinct isoforms of $\mathrm{HO}$ have been identified: HO-1 and HO-2. HO-2 is constitutively expressed and $\mathrm{HO}-1$ is an inducible form that is upregulated in response to oxidative stress, exerting cytoprotective effects against oxidative insults [3].

It has been shown that upregulation of HO-1 increases adiponectin secretion by remodeling adipose tissue in obese and diabetic rats [4-6]. These proteins function synergistically in modulating the metabolic syndrome phenotype, and the association between them has led to the proposal of the existence of an HO-1/adiponectin axis [7-9]. Adiponectin, a cytokine secreted by adipose tissue, has been shown to possess a vascular protective role by preserving endothelial cell function through activating the AMP-activated kinase (AMPK)-phosphatidylinositol 3-kinase (PI3K)/Akt-endothelial nitric oxide synthase (eNOS) pathway and increasing nitric oxide (NO) production in obese and diabetic subjects $[10,11]$. Our previous study also found that HO-1 induction with CoPP improves FFA-induced endothelial dysfunction in the rat aorta by activation of the AMPK-PI3K-eNOS pathway as a result of increased adiponectin levels [12]. However, it is still unclear whether upregulation of HO-1 has a direct beneficial effect on endothelial function in obesity besides the effects via regulating adiponectin levels. Therefore, in this study, we investigated whether upregulation of HO-1 directly improves endothelial function independent of adiponectin changes in obese rats and, if so, the potential mechanisms involved. Therefore, we used cobalt protoporphyrin (CoPP) to induce HO-1 and examined the effects of CoPP on endothelial function in the thoracic aorta isolated from high-fat diet-induced obese rats in vitro. We also examined the effects of the AMPK, PI3K and eNOS inhibitors on endothelial function. To verify that the effects of CoPP were because of increased HO-1 activity, we also treated the aorta concurrently with stannous protoporphyrin (SnPP) to inhibit HO-1 activity.

\section{Materials and Methods}

\section{Materials}

CoPP and SnPP were purchased from Frontier Scientific, Inc. (Logan, UT, USA). Norepinephrine (NE), acetylcholine (Ach), sodium nitroprusside (SNP), nitro-L-arginine methyl ester (L-NAME) and Compound C were purchased from Sigma (St. Louis, MO, USA). Antibodies for western blot analysis were obtained from Santa Cruz Biotechnology (Santa Cruz, CA, USA). Adiponectin and high-sensitivity C-reactive protein (hsCRP) assay kits were purchased from Uscn Life Science Inc. (Wuhan, China). H0-1 assay kit was purchased from Stressgen-Assay Design (Ann Arbor, MI, USA). NO and malondialdehyde (MDA) assay kits were purchased from Beyotime Biotechnology (Beijing, China). Serum TNF- $\alpha$ enzyme-linked immunosorbent assay (ELISA) kit was purchased from R\&D Systems (Minneapolis, MN, USA). Serum levels of adiponectin and hs-CRP were measured by ELISA (Uscn Life Science Inc.).

\section{Experimental animals}

All experimental animals were housed and received approval of the Institutional Animal Care and Use Committee (IACUC). The study conformed to the Guide for Care and Use of Laboratory Animals published in the Belgian Regulations. Eight weeks old male Wistar rats (specific pathogen-free quality) were purchased from Shandong Lukang Pharmaceutical Limited Company (Jining, China). All rats were randomly divided into a normal control (NC) group and an obesity (OB) group. The NC group was fed a regular diet (330 $\mathrm{kcal} / 100 \mathrm{~g}$ ) and the OB group was fed a high-fat diet (493 kcal/100 g) [13]. All rats were housed under standard laboratory conditions with free access to water and food for 10 weeks. Body weight and food intake were monitored weekly. After 10 weeks, the rats were anesthetized with an intraperitoneal injection of sodium pentobarbital $(60 \mathrm{mg} / \mathrm{kg}$ ). The blood was collected from the heart, and the visceral fat (VF including epididymal fat, perirenal fat and mesenteric fat) was harvested and weighed.

\section{KARGER}




\section{Cellular Physiology Cell Physiol Biochem 2015;36:1480-1490

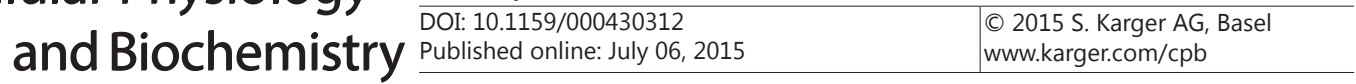 \\ Han et al.: Ho-1 Improves Endothelial Function}

\section{Evaluation of endothelial function}

Next, the intact thoracic aorta was immediately dissected, immersed in Krebs-Henseleit bicarbonate buffer (K-H solution: $118.3 \mathrm{mM} \mathrm{NaCl}, 4.7 \mathrm{mM} \mathrm{KCl}, 1.2 \mathrm{mM} \mathrm{KH}_{2} \mathrm{PO}_{4}, 1.2 \mathrm{mM} \mathrm{MgSO}_{4}, 25 \mathrm{mM} \mathrm{NaHCO}_{3}, 2.5 \mathrm{mM}$ $\mathrm{CaCl}_{2}, 5.5 \mathrm{mM}$ dextrose) free of adherent fat and connective tissue and cut into 3-mm long rings to measure endothelial function, as described previously [13-15]. Then, the vascular rings were mounted on two steel wires and immersed in individual organ chambers filled with $20 \mathrm{~mL}$ of $\mathrm{K}-\mathrm{H}$ solution at $37^{\circ} \mathrm{C}$ and linked with a force transducer (PowerLab, ADInstruments, Castle Hill, NSW, Australia). To study the direct effects of HO-1 upregulation on the vascular function of obese rats, vascular segments of control rats and obese rats were incubated in an organ bath in the presence of CoPP $(20 \mathrm{uM})$ or CoPP plus SnPP (20 uM $)$. The rings were equilibrated for $60 \mathrm{~min}$ with a preload of $1.0 \mathrm{~g}$. Tissues were washed every $30 \mathrm{~min}$ with K-H solution. Subsequently, all rings were first contracted with $\mathrm{KCl}(60 \mathrm{mM})$ and rinsed several times in $\mathrm{K}-\mathrm{H}$ solution. Before addition of Ach $\left(10^{-8}\right.$ to $\left.10^{-4} \mathrm{~mol} / \mathrm{L}\right)$ or SNP $\left(10^{-8}\right.$ to $\left.10^{-4} \mathrm{~mol} / \mathrm{L}\right)$ to the solution for assessment of the endothelium-dependent vasodilatation (EDV) and endothelium-independent vasodilatation (EIV) of the rings, $\mathrm{NE}(1 \mu \mathrm{M})$ was added to induce pre-contraction. The collected data were used to prepare concentration-relaxation response curves.

\section{Plasma measurements}

The plasma glucose level was measured by the glucose oxidase method. The plasma free fatty acids (FFA) level and serum lipids profile including total cholesterol (TC), triglycerides (TG), and malonic dialdehyde (MDA) were measured with colorimetric assays. Serum levels of TNF- $\alpha$, hs-CRP and adiponectin were measured by ELISA.

\section{Total NO production and eNOS activity measurement}

Total NO production (the concentration of nitrite and nitrate) by aortic segments was determined by a modified Griess method, as described previously [16, 17]. Briefly, after Ach $\left(10^{-4} \mathrm{M}\right)$ was added, $100 \mu \mathrm{l}$ of $\mathrm{K}-\mathrm{H}$ solution taken from the bath were mixed with $100 \mu \mathrm{l}$ of modified Griess reagent. After incubation at room temperature, the NO concentration was spectrophotometrically determined at $540 \mathrm{~nm}$ according to the manufacturer's instructions. The values were estimated per amount of dry weight of the tissue. Endothelial eNOS activity was determined by measuring the conversion of L-arginine to L-citrulline as described previously [18].

\section{HO activity, HO-1 concentration and superoxide anion measurement}

After the organ bath experiment, HO activity was assayed in aortic homogenates as described previously. Briefly, spectrophotometry was used to measure the concentration of bilirubin, the end product of heme degradation, by calculating the difference in absorption between 464 and $530 \mathrm{~nm}$. HO-1 concentration was determined by ELISA according to the manufacturer's instructions. Aortic superoxide anion production was measured using the lucigenin chemiluminescence method as described previously [19]. Superoxide production was expressed as counts per minute per milligram aortic tissue.

\section{Immunohistochemistry}

After the organ bath experiment, the aorta was fixed in formalin and used for nuclear transcription factor kappa B (NF- $\mathrm{B}$ p65) immunohistochemistry. Sections $(5 \mu \mathrm{m})$ were deparaffinized in xylene, rehydrated in ethanol, and then quenched in $3 \% \mathrm{H}_{2} \mathrm{O}_{2}$ solution for $10 \mathrm{~min}$ at $37^{\circ} \mathrm{C}$ before restoring antigen retrieval. The sections were then incubated with the NF- $\mathrm{B}$ p 65 antibody followed by a secondary antibody. The slides were stained with 3,3'-diaminobenzidine and counterstained with hematoxylin. The number of $\mathrm{NF}-\kappa \mathrm{B}$ p 65 positive nuclei and cells was analyzed using Image-Pro plus 6.0 software.

\section{Western blot analysis}

Equal amounts of total protein extracts from the endothelial cells were electrophoresed in 10\% SDSPAGE, probed with different primary antibodies (anti-AMPK, anti-p-AMPK, anti-Akt, anti-p-Akt, anti-eNOS and anti-p-eNOS, Ser 1177), and then incubated with the appropriate secondary antibodies. The protein expression levels were quantified using the Pro-plus 6.0 image-processing system. 
In vitro and signaling pathway studies

To investigate whether the AMPK-PI3K/Akt-eNOS pathway is required for the effect of CoPP on EDV in obese rats, the aortic rings obtained from obese rats were incubated in an organ bath containing K-H solution with CoPP in the presence or absence of the following specific inhibitors of candidate pathways: (1) $20 \mu \mathrm{M}$ AMPK-specific inhibitor, compound C, which competes with ATP-binding sites (2) $30 \mu \mathrm{M}$ PI3K inhibitor, LY294002[20], which inhibits PI3K activity via competitive inhibition of an ATP binding site on the p85 $\alpha$ subunit; (3) $10^{-4}$ M, L-NAME (Sigma), an analog of arginine that inhibits NO production. After 60 min incubation, the aortic rings were pre-contracted with NE $(1 \mu \mathrm{M})$, followed by Ach $\left(10^{-8}-10^{-4} \mathrm{M}\right)$ or SNP $\left(10^{-8}-10^{-4} \mathrm{M}\right)$ to determine EDV and EIV.

\section{Statistical analysis}

All data were subjected to one-way ANOVA statistical analysis using the SPSS 16.0 statistical package, and data are expressed as mean \pm SD. The Student-Newman-Keuls method was used to detect significant differences in the intergroup comparisons. $p<0.05$ was considered statistically significant.

\section{Results}

\section{Biometric and blood parameters of rats}

After 10 weeks, the rats in the OB group exhibited increased body weight, visceral fat and visceral fat index (visceral fat/body weight), compared with the rats in the NC group (Table 1). No significant difference was found in the plasma glucose between the two groups $(p>0.05)$. As expected, the plasma FFA levels and the serum levels of hs-CRP and MDA were elevated, and the serum adiponectin levels were lower in the OB group compared with those in the NC group $(p<0.05)$ (Table 2$)$.

\section{Effect of HO-1 induction on $\mathrm{HO}$ activity and HO-1 concentration}

$\mathrm{HO}$ activity and HO-1 concentration in the aorta were measured (Fig. 1). We observed a notable decrease in $\mathrm{HO}$ activity and $\mathrm{HO}-1$ concentration in the obese rats compared with those in the control rats $(p<0.05)$. However, induction of HO-1 with CoPP increased HO

Table 1. Biometric parameters of Rats in the two Groups ( $\mathrm{n}=8)$. Data are shown as mean $\pm \mathrm{SD}^{*} \mathrm{P}<0.01$ vs

\begin{tabular}{cccc}
\hline Group & $\begin{array}{c}\text { Weight } \\
(\mathrm{g})\end{array}$ & $\begin{array}{c}\text { Visceral fat } \\
(\mathrm{g})\end{array}$ & Visceral fat/ weight $\left(10^{-3}\right)$ \\
\hline $\mathrm{NC}$ & $349.1 \pm 18.6$ & $9.41 \pm 1.31$ & $27.01 \pm 4.09$ \\
OB & $414.4 \pm 24.9^{*}$ & $27.94 \pm 3.47^{*}$ & $67.83 \pm 11.01^{*}$ \\
\hline
\end{tabular}

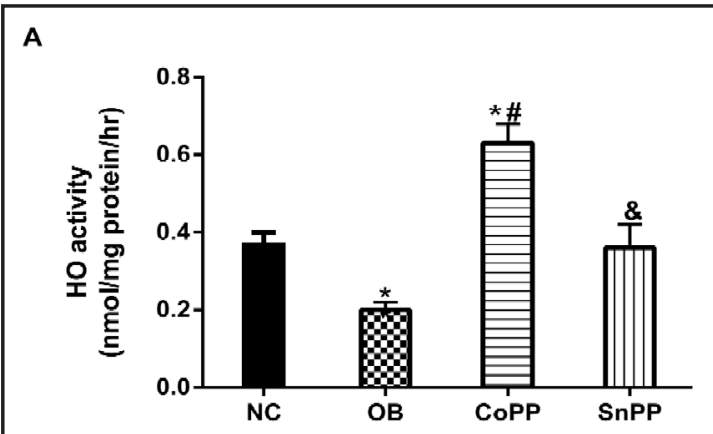

B

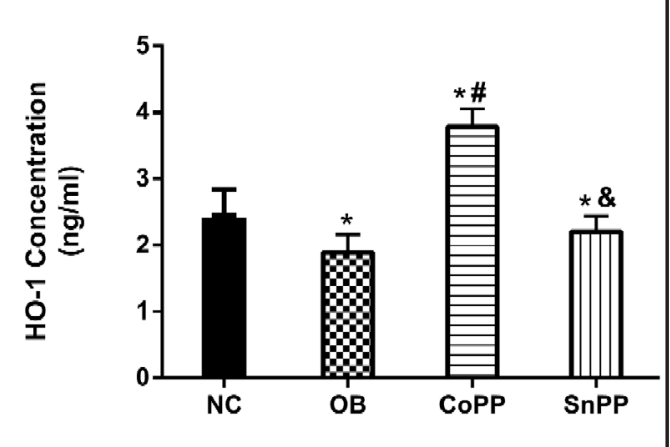

Fig. 1. HO activity (A) and HO-1 concentration (B) of the aorta 'from the studies groups. $n=7-8$ vascular segments/group from 7-8 rats. NC, aorta rings from the control rats; $\mathrm{OB}$, aorta rings from the obese rats; CoPP, aorta rings from the obese rats incubated with CoPP; SnPP, aorta rings from the obese rats co-incubated with CoPP and SnPP. Data are shown as mean \pm SD. ${ }^{*} \mathrm{P}<0.05 v s$ NC group; ${ }^{\#} \mathrm{P}<0.05$ vs OB group; ${ }^{\text {P }}<$ 0.05 vs CoPP group.

\section{KARGER}


Table 2 Blood parameters of Rats in the two Groups $(n=8)$. Data are shown as mean \pm SD. TG, triglyceride; FFA, free fatty acids; MDA, malondialdehyde; hs-CRP, high-sensitivity c-reactive protein; ${ }^{*} \mathrm{P}<0.01$ vs NC group

\begin{tabular}{ccccccc}
\hline Group & Glucose $(\mathrm{mmol} / \mathrm{L})$ & $\begin{array}{c}\text { TG } \\
(\mathrm{mmol} / \mathrm{L})\end{array}$ & $\begin{array}{c}\text { FFA } \\
(\mathrm{mmol} / \mathrm{L})\end{array}$ & $\begin{array}{c}\text { hs-CRP } \\
(\mathrm{mg} / \mathrm{L})\end{array}$ & MDA (umol/L) & $\begin{array}{c}\text { Adiponectin } \\
(\mathrm{ug} / \mathrm{ml})\end{array}$ \\
\hline NC & $5.81 \pm 0.43$ & $0.51 \pm 0.16$ & $0.38 \pm 0.10$ & $0.91 \pm 0.23$ & $1.36 \pm 0.24$ & $19.21 \pm 1.18$ \\
OB & $6.02 \pm 0.51$ & $1.32 \pm 0.37^{*}$ & $1.01 \pm 0.21^{*}$ & $2.68 \pm 0.51^{*}$ & $4.42 \pm 0.38^{*}$ & $15.01 \pm 1.36^{*}$ \\
\hline
\end{tabular}

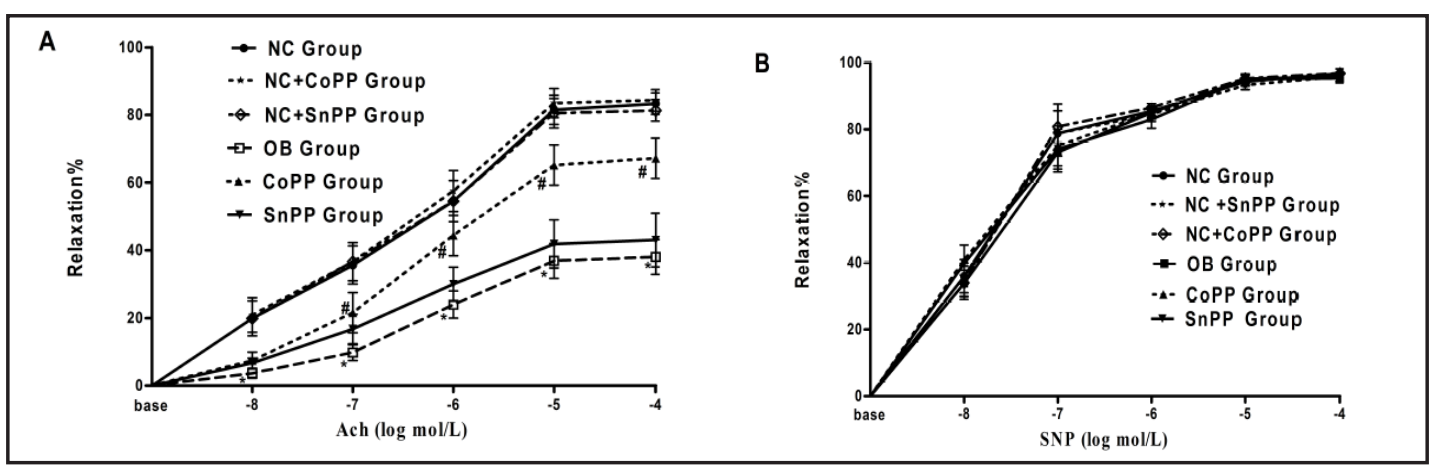

Fig. 2. Vasorelaxation response of thoracic aorta rings to Ach (A) and SNP(B) in the studied groups. Relaxation expressed as percentage of maximal NE-induced vasoconstriction. $n=7-8$ vascular segments/group from 7-8 rats. $\mathrm{NC}$, aorta rings from the control rats; $\mathrm{OB}$, aorta rings from the obese rats; $\mathrm{NC}+\mathrm{CoPP}$, aorta rings from the control rats incubated with CoPP; $\mathrm{NC}+\mathrm{SnPP}$, aorta rings from the control rats co-incubated with CoPP and SnPP; CoPP, aorta rings from the obese rats incubated with CoPP; SnPP, aorta rings from the obese rats co-incubated with CoPP and SnPP; Data are shown as mean \pm SD. *P $<0.05$ vs NC group. "P $<0.05$ vs OB group. ${ }^{\&} \mathrm{P}<0.05$ vs CoPP group.

activity and HO-1 concentration. These effects were abolished by co-incubated with SnPP $(p<0.05)$.

\section{Effect of HO-1 induction on Endothelial Function}

As expected, Ach-induced EDV was significantly attenuated in the obese rats, compared with the NC group $(p<0.05)$. However, this attenuation decreased when the ring segments of the obese rats were incubated with CoPP, and this effect was reversed by co-incubation with SnPP. When the ring segments of the control rats were incubated with CoPP or co-incubated with CoPP and SnPP, no significant differences in EDV were found. No significant differences in SNP-induced EIV were found between these groups (Fig. 2).

Effect of HO-1 induction on NO production, eNOS activity and superoxide anion production

To determine whether HO-1 upregulation increases endothelium-derived NO production, we measured NO levels in the absence or presence of CoPP. The NO concentration and eNOS activity were significantly lower in the obese rats compared with those in the control rats $(p<0.05)$. However, the NO levels and eNOS activity in the obese rats treated with CoPP substantially increased compared with those in the obese rats incubated in the absence of CoPP and in obese rats incubated in the present of SnPP $(p<0.05$; Fig. $3 \mathrm{~A}-\mathrm{B})$. In addition, the NO levels in the obese rats treated with CoPP were significant reduced when the aorta were pretreated with Compound C or LY294002 compared with those in the obese rats treated with CoPP $(p<0.05)$. We also observed an increase in superoxide anion production in the obese rats compared with those in the control rats $(p<0.05)$. However, induction of HO-1 with CoPP decreased superoxide anion production. These effects were abolished by co-incubated with SnPP ( $p<0.05$; Fig. 3 C). 
Fig. 3. Effect of HO-1 induction on total NO production (A), eNOS activity (B) and superoxide production (C) from the studies groups. $n=7-8$ vascular segments/group from 7-8 rats. $\mathrm{NC}$, aorta rings from the control rats; $\mathrm{OB}$, aorta rings from the obese rats; CoPP, aorta rings from the obese rats incubated with CoPP; SnPP, aorta rings from the obese rats co-incubated with CoPP and SnPP. Data are shown as mean \pm SD. ${ }^{*} \mathrm{P}<$ 0.05 vs NC group. ${ }^{\#} \mathrm{P}<0.05$ vs $\mathrm{OB}$ group. ${ }^{\&} \mathrm{P}<$ 0.05 vs CoPP group.

Effect of $\mathrm{HO}-1$ induction on $\mathrm{NF}-\kappa \mathrm{B}$ p 65 expression

A

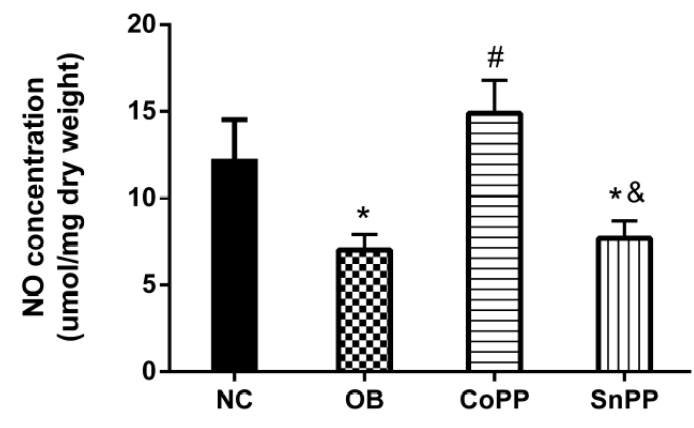

B

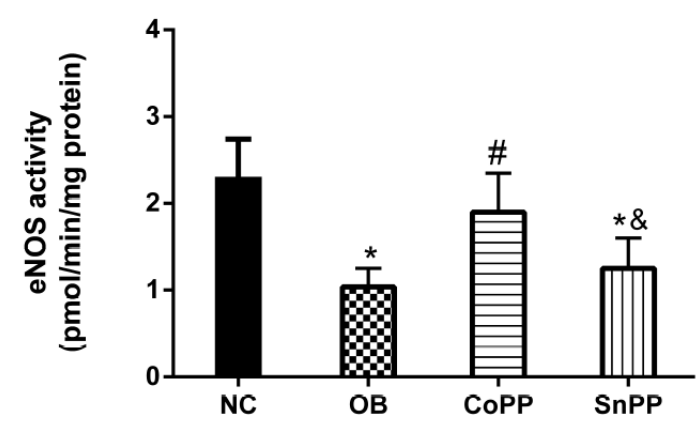

C

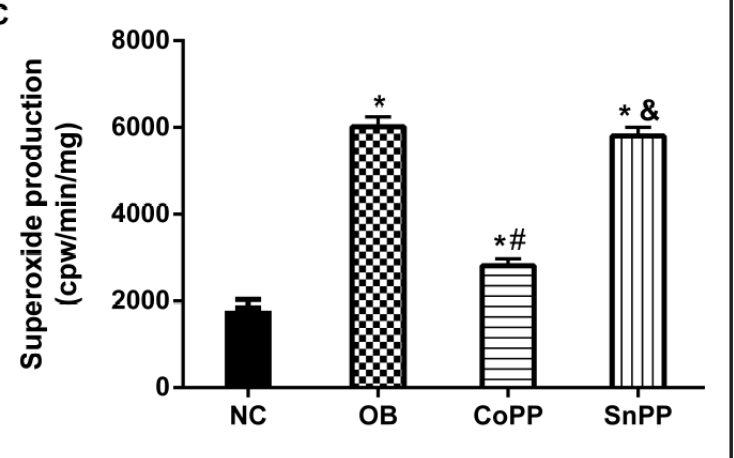

Immunohistochemistry showed increased NF- $\kappa \mathrm{B}$ p65 expression in the aorta of obese rats compared with that in the control rats $(p<0.05)$. However, upregulation of HO-1 with

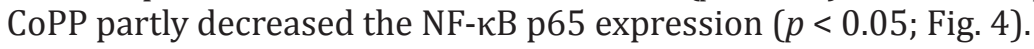

Effect of HO-1 induction on the AMPK-PI3K/Akt-eNOS pathway in endothelial cells

To investigate the mechanism of HO-1 upregulation in modulating vascular function in obese rats, total and phosphorylated AMPK, Akt and eNOS expression was examined. As shown in Figure 5, upregulation of HO-1 with CoPP increased the phosphorylation of AMPK, Akt and eNOS in obese rats $(p<0.05)$. However, no significant differences were observed when the rats were co-treated with CoPP and SnPP ( $p>0.05)$.

\section{Signaling pathway studies}

As shown above, pre-incubation of vessels from obese rats with CoPP significantly increased EDV, compared with the control rats. However, this beneficial effect of CoPP was partly attenuated in the presence of $20 \mu \mathrm{M}$ compound C, $30 \mu \mathrm{M} \mathrm{LY} 294002$, and $10^{-4} \mathrm{M}$ L-NAME (Fig. 6). No statistical significance in EIV was observed among the different groups (data not shown). 
Fig. 4. Effect of HO-1 induction on $N F-\kappa B$ p65 expression of the aorta in the rats. $A$, aorta rings from the control rats; $B$, aorta rings from the obese rats; $\mathrm{C}$, aorta rings from the obese rats incubated with CoPP; D, aorta rings from the obese rats co-incubated with CoPP and SnPP. Magnifications: $\times 100$.

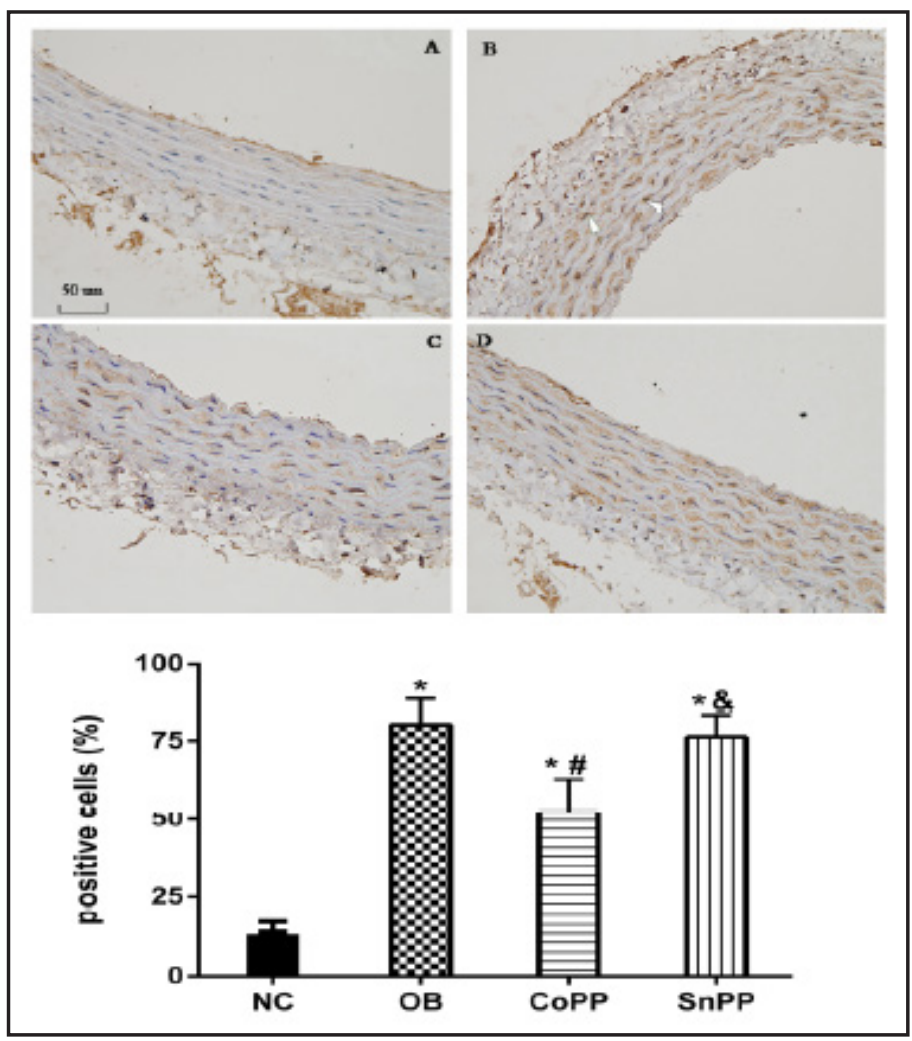

Fig. 5. Western blot analysis for the expressions of the AMPK, Akt and eNOS proteins in the endothelial cells. NC, aorta rings from the control rats; $\mathrm{OB}$, aorta rings from the obese rats; $\mathrm{CoPP}$, aorta rings from the obese rats incubated with CoPP; SnPP, aorta rings from the obese rats co-incubated with CoPP and SnPP. *P < 0.05vs NC group; \# $\mathrm{P}<0.05$ vs OB group; ${ }^{\&} \mathrm{P}<0.05$ vs CoPP group.

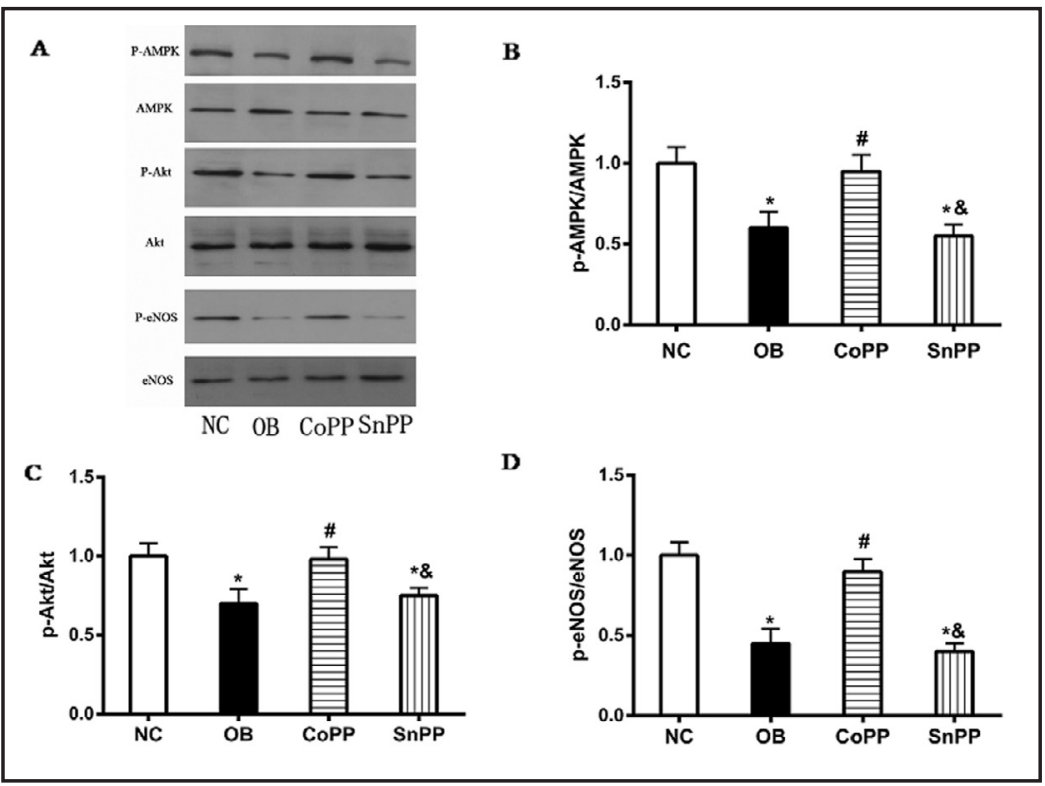

\section{Discussion}

This study demonstrated that induction of HO-1 with CoPP could directly restore the impaired aortic endothelial function in diet-induced obese rats. The mechanism of this protective effect is related to increasing NO production, independent of adiponectin changes, as well as reducing inflammation and oxidative stress. Moreover, CoPP significantly increased eNOS phosphorylation. The results showed that the protective effect of HO-1 induction was mediated, at least in part, by the activation of the AMPK-PI3K/Akt-eNOS signaling pathway. These results suggest that $\mathrm{HO}-1$ induction plays an important role in modulating endothelial function in vitro. 
Fig. 6. Vasorelaxation response of thoracic aorta segment to Ach in the studied groups. The aortic rings obtained from obese rats were incubated with CoPP in the presence or absence of 20 $\mu \mathrm{M}$ AMPK inhibitor (compound C), 30 $\mu \mathrm{M}$ PI3K inhibitor (LY294002) and $10^{-4}$ M eNOS inhibitor (L-NAME). $n=6-8$ vascular segments/group from 6-8 rats. Data are shown as mean \pm SD. NC, aorta rings from the control rats; $\mathrm{OB}$, aorta rings from the obese rats; CoPP, aorta rings from the obese rats incubated with CoPP; ${ }^{*} \mathrm{P}<0.05$ vs NC group. ${ }^{*} \mathrm{P}<0.05$ vs OB group. ${ }^{\&} \mathrm{P}<0.05$ vs CoPP group.

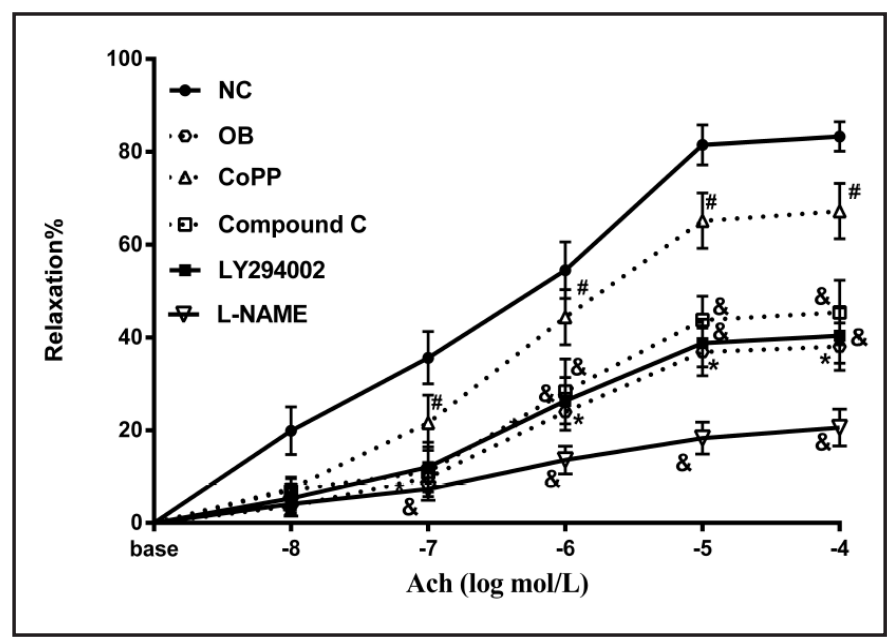

It has been well established that obesity is an independent risk factor for cardiovascular disease. Integrated endothelium plays a critical role in protecting the vasculature against atherosclerosis. Endothelial dysfunction, mainly endothelial-dependent vasorelaxation, has been observed in obesity-associated metabolic syndrome [10,21]. In our study, EDV was significantly impaired in diet-induced obese rats, but the EIV response to SNP did not change, which is consistent with previous studies $[13,14,22]$. Several risk-independent factors have been shown to be involved in the mechanism by which obesity influences endothelial dysfunction, such as high FFA levels, low adiponectin levels, chronic inflammation and oxidative stress, all of which impair endothelial NO production [10,23]. In this study, we also found that in diet-induced obese rats compared with control rats, abdominal adiposity was obvious, plasma FFA levels and serum levels of hs-CRP and MDA were elevated, and serum adiponectin levels were lower, indicating a successful obesity model.

HO-1, a stress response protein, can be induced by various oxidative insults. Numerous studies have shown that HO-1 induction is an important cellular protective mechanism against oxidative injury in obesity and diabetes-induced cardiovascular disease [24, 25]. H0-1 induction increased circulating adiponectin levels by remodeling adipose tissue in obese and diabetic rats $[4,5]$, and the association between these proteins has led to the proposal of the existence of an HO-1/adiponectin axis $[8,9]$. It has been shown that HO-1 induction could improve endothelial function by increasing circulating adiponectin levels through the HO-1/adiponectin axis, which may independently protect against endothelial dysfunction in obesity through activation of the AMPK/PI3K/eNOS pathway and NO production [10, 26]. However, it is still unclear whether HO-1 induction has a direct beneficial effect on endothelial function in obesity besides the effects involving regulation of adiponectin levels. In our study, incubation of the ring segments of the obese rats with CoPP, an HO- 1 inducer, significantly improved Ach-induced EDV in vitro, and this effect was reversed by incubation with the HO-1 inhibitor, SnPP. These results suggest that induction of HO-1 could directly restore impaired aortic endothelial function in high-fat diet-induced obese rats, independent of increasing adiponectin levels.

It is known that impaired EDV in obese rats arises from decreased production and/ or bioactivity of NO induced by eNOS phosphorylation $[15,27]$. In the present study, we found that induction of HO-1 with CoPP significantly enhanced eNOS phosphorylation and total production of $\mathrm{NO}$ by thoracic aortic rings compared with that by rings from obese rats without CoPP pre-incubation. These changes were not observed when the aortic rings were incubated with the HO-1 inhibitor, SnPP. In addition, this CoPP beneficial effect on endothelial function was partly attenuated when the eNOS inhibitor, L-NAME, was added in vitro. These results suggest that HO-1 induction could directly improve endothelial function through the eNOS-NO signaling pathway. 


\section{Cellular Physiology Cell Physiol Biochem 2015;36:1480-1490 \begin{tabular}{l|l} 
and Biochemistry Published online: July 06, 2015 & $\begin{array}{l}\text { C) 2015 S. Karger AG, Basel } \\
\text { www.karger.com/cpb }\end{array}$ \\
\hline
\end{tabular} \\ Han et al.: Ho-1 Improves Endothelial Function}

This study also sought to determine the possible mechanism that underlies the relationship between $\mathrm{HO}-1$ induction and the eNOS-NO signaling pathway in the endothelium. It is known that the HO-1/adiponectin axis can regulate eNOS-NO through the AMPK-PI3K/ Akt signaling pathway [28]. However, whether induction of HO-1 can directly regulate eNOSNO by activating AMPK-PI3K/Akt in vitro independent of adiponectin is still unknown. As adiponectin cannot be secreted from aorta rings, we incubated the aorta with CoPP in vitro and found that induction of HO-1 with CoPP increased the phosphorylation of AMPK and Akt in the aorta of obese rats. However, no significant differences were observed when the aorta was treated with SnPP. In addition, the beneficial effect of HO-1 induction on EDV was partly attenuated in the presence of AMPK inhibitor, PI3K inhibitor or eNOS inhibitor in vitro. In addition, the NO levels in the obese rats treated with CoPP were significant reduced when the aorta were pretreated with AMPK inhibitor or PI3K inhibitor compared with those in the obese rats treated with CoPP. These results suggest that HO-1 induction can also promote NO production through the AMPK-PI3K/Akt-eNOS pathway independent of adiponectin.

$\mathrm{NF}-\kappa \mathrm{B}$ is a group of inducible transcription factors and proinflammatory master switch that controls the production of a host of inflammatory factors [29]. NF- $\mathrm{BB}$ p65 is a subunit of NF- $\kappa \mathrm{B}$ transcription complex, which plays a crucial role in inflammatory responses and is responsible for the strong transcription activating potential of NF- $\kappa \mathrm{B}$. The activation of the $\mathrm{NF}-\kappa \mathrm{B}$ is then translocated to the nucleus, where it binds to specific sequences of DNA and induces the expression of many inflammatory cytokines. Both inflammation and oxidative stress could reduce NO production by enhancing the oxidation of tetrahydrobiopterin, a critical cofactor of eNOS, in obesity [14]. When there is a tetrahydrobiopterin deficiency, L-arginine produces superoxides rather than NO by eNOS uncoupling, which further reduces NO production [30]. In addition, overproduction of superoxide anions induced by FFA and inflammation leads to oxidative stress, a harmful process resulting in cellular structural damage, which can also quench NO to form peroxynitrite in obesity [14, 31]. In this study, we found that HO-1 induction with CoPP reduced superoxide anion production and NF- $\mathrm{KB}$ p65 expression in the aorta of obese rats, and that these effects were abolished by SnPP. Together, these results suggest that HO-1 induction can also promote NO production by reducing inflammation and oxidative stress.

In this study, induction of HO- 1 with CoPP directly restored impaired aortic endothelial function in obese rats. However, previous studies have demonstrate that transgenic mice overexpressing HO-1 in vascular smooth muscle cells exhibit worsening not improvement of vascular relaxation [32]. Similarly, mice chronically treated in vivo with CoPP also had worsening of vascular relaxation [33]. It is possible that administering CoPP via incubation of the vessel in a bath solution in vitro may have different effects on vascular relaxation compared with chronic induction of HO-1 in vivo. A possible explanation for this contradiction may be associated with the various actions of the by-products of HO-1 induction on endothelial function under different conditions. However, further studies will be required to elucidate the cytoprotective mechanisms of HO-1 induction on endothelial function.

In summary, this study demonstrated that induction of $\mathrm{HO}-1$ with CoPP directly restored impaired aortic endothelial function in obese rats. The mechanism of this protective effect is related to enhanced NO production, in a manner independent of adiponectin changes, and to reduced inflammation and oxidative stress. The molecular pathways appear to involve, at least in part, the activation of the AMPK-eNOS signaling pathway. These results suggest that besides the HO-1/adiponectin axis, HO-1 induction plays an important role in modulating endothelial function in obesity.

\section{Acknowledgements}

This work was supported by the National Natural Science Foundation of China (Grants NO. 81300688 and NO. 81400829), and the Science and Technology Innovation Fund of Weifang Medical University (NO. K1301021, NO. K1302017).

\section{KARGER}




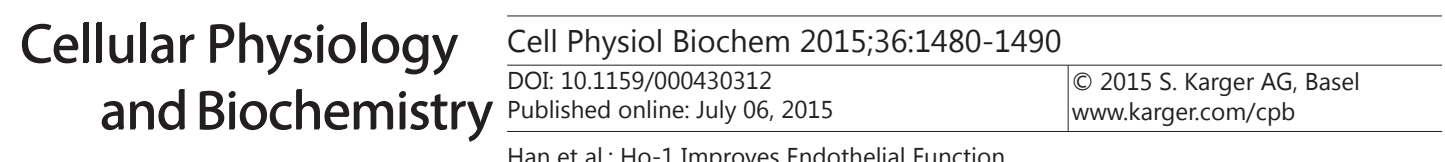

We would like to thank the Edanz group China for providing language editing assistance for our manuscript.

\section{Disclosure Statement}

The authors declare no conflict of interest.

\section{References}

1 Bakhautdin B, Das D, Mandal P, Roychowdhury S, Danner J, Bush K, Pollard K, Kaspar JW, Li W, Salomon RG, McMullen MR, Nagy LE: Protective role of HO-1 and carbon monoxide in ethanol-induced hepatocyte cell death and liver injury in mice. J Hepatol 2014;61:1029-1037.

2 Sun Y, Li QF, Zhang Y, Hu R, Jiang H: Isoflurane preconditioning increases survival of rat skin randompattern flaps by induction of HIF-1alpha expression. Cell Physiol Biochem 2013;31:579-591.

3 Bauer M, Huse K, Settmacher U, Claus RA: The heme oxygenase-carbon monoxide system: regulation and role in stress response and organ failure. Intensive Care Med 2008;34:640-648.

4 Li M, Kim DH, Tsenovoy PL, Peterson SJ, Rezzani R, Rodella LF, Aronow WS, Ikehara S, Abraham NG: Treatment of obese diabetic mice with a heme oxygenase inducer reduces visceral and subcutaneous adiposity, increases adiponectin levels, and improves insulin sensitivity and glucose tolerance. Diabetes 2008;57:1526-1535.

5 Kim DH, Burgess AP, Li M, Tsenovoy PL, Addabbo F, McClung JA, Puri N, Abraham NG: Heme oxygenasemediated increases in adiponectin decrease fat content and inflammatory cytokines tumor necrosis factor-alpha and interleukin-6 in Zucker rats and reduce adipogenesis in human mesenchymal stem cells. J Pharmacol Exp Ther 2008;325:833-840.

6 L'Abbate A, Neglia D, Vecoli C, Novelli M, Ottaviano V, Baldi S, Barsacchi R, Paolicchi A, Masiello P, Drummond GS, McClung JA, Abraham NG: Beneficial effect of heme oxygenase-1 expression on myocardial ischemia-reperfusion involves an increase in adiponectin in mildly diabetic rats. Am J Physiol Heart Circ Physiol 2007;293:H3532-3541.

$7 \quad$ Li M: Treatment of obese diabetic mice with a heme oxygenase inducer reduces visceral and subcutaneous adiposity, increases adiponectin levels, and improves insulin sensitivity and glucose tolerance. Diabetes 2008;57:1526-1535.

8 Kim DH: Epoxyeicosatrienoic Acid Agonist Regulates Human Mesenchymal Stem Cell-Derived Adipocytes Through Activation of HO-1-pAKT Signaling and a Decrease in PPAR . Stem Cells Dev 2010;19:1863-1873.

9 Cao J: High fat diet enhances cardiac abnormalities in SHR rats: Protective role of heme oxygenaseadiponectin axis. Diabetol Metab Syndr 2011;3:37.

10 Wang B, Yu Y, Han L: Adiponectin improves endothelial dysfunction caused by elevated FFAs levels, partially through cAMP-dependent pathway. Diabetes Res Clin Pract 2012;97:119-124.

11 Hopkins TA, Ouchi N, Shibata R, Walsh K: Adiponectin actions in the cardiovascular system. Cardiovasc Res 2007;74:11-18.

12 Han F, Hui Z, Zhang S, Hou N, Wang Y, Sun X: Induction of haemeoxygenase-1 improves FFA-induced endothelial dysfunction in rat aorta. Cell Physiol Biochem 2015;35:1230-1240.

13 Hou N, Han F, Wang M, Huang N, Zhao J, Liu X, Sun X: Perirenal fat associated with microalbuminuria in obese rats. Int Urol Nephrol 2014;46:839-845.

14 Sun X, Yu Y, Han L: High FFA levels related to microalbuminuria and uncoupling of VEGF-NO axis in obese rats. Int Urol Nephrol 2013;45:1197-1207.

15 Hou N, Huang N, Han F, Zhao J, Liu X, Sun X: Protective effects of adiponectin on uncoupling of glomerular VEGF-NO axis in early streptozotocin-induced type 2 diabetic rats. Int Urol Nephrol 2014;46:2045-2051.

16 Li R, Wang WQ, Zhang H, Yang X, Fan Q, Christopher TA, Lopez BL, Tao L, Goldstein BJ, Gao F, Ma XL: Adiponectin improves endothelial function in hyperlipidemic rats by reducing oxidative/nitrative stress and differential regulation of eNOS/iNOS activity. Am J Physiol Endocrinol Metab 2007;293:E1703-1708.

17 Kono T, Saito M, Kinoshita Y, Satoh I, Shinbori C, Satoh K: Real-time monitoring of nitric oxide and blood flow during ischemia-reperfusion in the rat testis. Mol Cell Biochem 2006;286:139-145. 


\section{Cellular Physiology Cell Physiol Biochem 2015;36:1480-1490

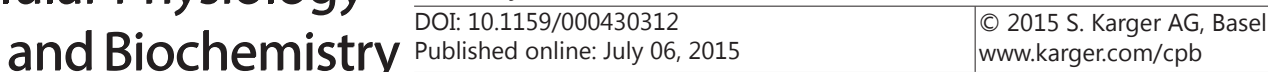 \\ Han et al.: Ho-1 Improves Endothelial Function}

18 Jiang X, Yang F, Tan H, Liao D, Bryan RM Jr, Randhawa JK, Rumbaut RE, Durante W, Schafer AI, Yang X, Wang H: Hyperhomocystinemia impairs endothelial function and eNOS activity via PKC activation. Arterioscler Thromb Vasc Biol 2005;25:2515-2521.

19 Gupte SA, Kaminski PM, Floyd B, Agarwal R, Ali N, Ahmad M, Edwards J, Wolin MS: Cytosolic NADPH may regulate differences in basal Nox oxidase-derived superoxide generation in bovine coronary and pulmonary arteries. Am J Physiol Heart Circ Physiol 2005;288:H13-21.

20 Qiu C, Xie Q, Zhang D, Chen Q, Hu J, Xu L: GM-CSF induces cyclin D1 expression and proliferation of endothelial progenitor cells via PI3K and MAPK signaling. Cell Physiol Biochem 2014;33:784-795.

21 Sun X, Han F, Miao W, Hou N, Cao Z, Zhang G: Sonographic evaluation of para- and perirenal fat thickness is an independent predictor of early kidney damage in obese patients. Int Urol Nephrol 2013;45:1589-1595.

22 Sun XD, Yu YR: The protective effects of rosiglitazone on kidney in diet-induced obese rats. Sichuan Da Xue Xue Bao Yi Xue Ban 2014; 45:24-28, 33.

23 Han F, Hou N, Miao W, Sun X: Correlation of ultrasonographic measurement of intrarenal arterial resistance index with microalbuminuria in nonhypertensive, nondiabetic obese patients. Int Urol Nephrol 2013;45:1039-1045.

24 Duann P: GEC-targeted HO-1 expression reduces proteinuria in glomerular immune injury. AM J PhysiolRenal Physiol 2009;297:F629.

25 Calay D, Mason JC: The multifunctional role and therapeutic potential of HO-1 in the vascular endothelium. Antioxid Redox Signal 2014;20:1789-1809.

26 Pansuria M, Xi H, Li L, Yang XF, Wang H: Insulin resistance, metabolic stress, and atherosclerosis. Front Biosci (Schol Ed) 2012;4:916-931.

27 Wang XM, Song SS, Xiao H, Gao P, Li XJ, Si LY: Fibroblast growth factor 21 protects against high glucose induced cellular damage and dysfunction of endothelial nitric-oxide synthase in endothelial cells. Cell Physiol Biochem 2014;34:658-671.

28 Morello F, Perino A, Hirsch E: Phosphoinositide 3-kinase signalling in the vascular system. Cardiovasc Res 2009;82:261-271.

29 Sun X, Han F, Yi J, Han L, Wang B: Effect of Aspirin on the Expression of Hepatocyte NF-kappaB and Serum TNF-alpha in Streptozotocin-Induced Type 2 Diabetic Rats. J Korean Med Sci 2011;26:765-770.

30 Satoh M, Fujimoto S, Arakawa S, Yada T, Namikoshi T, Haruna Y, Horike H, Sasaki T, Kashihara N: Angiotensin II type 1 receptor blocker ameliorates uncoupled endothelial nitric oxide synthase in rats with experimental diabetic nephropathy. Nephrol Dial Transplant 2008;23:3806-3813.

31 Chen W, Zheng G, Yang S, Ping W, Fu X, Zhang N, Wang DW, Wang J: CYP2J2 and EETs Protect against Oxidative Stress and Apoptosis in Vivo and in Vitro Following Lung Ischemia/Reperfusion. Cell Physiol Biochem 2014;33:1663-1680.

32 Imai T, Morita T, Shindo T, Nagai R, Yazaki Y, Kurihara H, Suematsu M, Katayama S: Vascular smooth muscle cell-directed overexpression of heme oxygenase- 1 elevates blood pressure through attenuation of nitric oxide-induced vasodilation in mice. Circ Res 2001;89:55-62.

33 Stec DE, Vera T, McLemore GR Jr, Kelsen S, Rimoldi JM, Gadepalli RS, Ryan MJ: Heme oxygenase-1 induction does not improve vascular relaxation in angiotensin II hypertensive mice. Am J Hypertens 2008;21:189193. 